\title{
Early Detection of Emphysema Progression
}

\author{
Vladlena Gorbunova ${ }^{1}$, Sander S.A.M. Jacobs ${ }^{2}$, Pechin Lo ${ }^{1}$, Asger Dirksen ${ }^{3}$, \\ Mads Nielsen ${ }^{1,4}$, Alireza Bab-Hadiashar ${ }^{5}$, and Marleen de Bruijne ${ }^{1,6}$ \\ 1 Department of Computer Science, University of Copenhagen, Denmark \\ 2 Department of Biomedical Engineering, Eindhoven University of Technology, \\ The Netherlands \\ 3 Department of Respiratory Medicine, Gentofte University Hospital, Denmark \\ 4 Nordic Bioscience A/S, Herlev, Denmark \\ 5 Swinburne University of Technology, Hawthorn, Australia \\ ${ }^{6}$ Biomedical Imaging Group Rotterdam, Erasmus MC, Rotterdam, The Netherlands
}

\begin{abstract}
Emphysema is one of the most widespread diseases in subjects with smoking history. The gold standard method for estimating the severity of emphysema is a lung function test, such as forced expiratory volume in first second $\left(\mathrm{FEV}_{1}\right)$. However, several clinical studies showed that chest CT scans offer more sensitive estimates of emphysema progression. The standard CT densitometric score of emphysema is the relative area of voxels below a threshold (RA). The RA score is a global measurement and reflects the overall emphysema progression.

In this work, we propose a framework for estimation of local emphysema progression from longitudinal chest CT scans. First, images are registered to a common system of coordinates and then local image dissimilarities are computed in corresponding anatomical locations. Finally, the obtained dissimilarity representation is converted into a single emphysema progression score. We applied the proposed algorithm on 27 patients with severe emphysema with CT scans acquired five time points, at baseline, after 3 , after 12, after 21 and after 24 or 30 months. The results showed consistent emphysema progression with time and the overall progression score correlates significantly with the increase in RA score.
\end{abstract}

\section{Introduction}

Emphysema is one of the most common chronic obstructive pulmonary diseases [1. It is characterized by irreversible destruction of the lung parenchyma and usually caused by smoking [2].

In clinical practice, the severity of emphysema is commonly assessed using different lung function tests. Along with the lung function tests chest CT scans has been used for diagnosis of emphysema and detection of emphysema progression. The standard CT density scores, such as relative area (RA) below certain threshold, e.g. $-950 \mathrm{HU}$ or $-930 \mathrm{HU}$, and the n-th percentile density (nPD) of the lungs, were applied to estimate the emphysema progression 34. CT densitometry scores have shown to be more sensitive measures of emphysema progression than lung function tests [4.

T. Jiang et al. (Eds.): MICCAI 2010, Part II, LNCS 6362, pp. 193200, 2010.

(C) Springer-Verlag Berlin Heidelberg 2010 
One of the major drawbacks of the standard CT density scores is their dependency on the inspiratory level [56]. Another important drawback is the lack of sensitivity, since the emphysema progression could only be measured once the intensity of lung tissue decreases below the standard threshold. Texture analysis may resolve this problems. This issue was investigated in a recent study, where a texture-based classification approach was proposed as alternative to the standard emphysema scores [7. The results showed that the texture-based approach outperforms the RA scores in differentiating diseased from healthy subjects.

Several studies proposed how to estimate disease progression from longitudinal CT scans [56. Authors proposed a method where CT scans are first registered to a common framework and then emphysema progression is estimated based on the average intensity decrease between the two successive scans.

In this paper, we propose a more general way of assessing emphysema progression between a pair of images. First, images are registered to a common system of coordinates. Second, local image histograms at a given location are obtained and dissimilarity measures between the histograms are computed. Thirdly, a measure of progression at the given location is derived from the dissimilarity measures. Finally, an overall disease progression score between the two images is computed. This method is applied to detect emphysema progression in a longitudinal study of patients with Alpha-1 antitrypsin deficiency [4].

\section{Method}

In this section we describe in details the work flow of the algorithm. The first subsection (2.1) briefly describes the image registration method that was applied to establish the spatial correspondence between images. The following subsection (2.2) presents how local dissimilarities were constructed. The last subsection (2.3) describes how the local disease progression score on subject level was derived from the set of local dissimilarity measures.

\subsection{Registration}

The image registration framework presented in [6] is used to register the images to a common system of coordinates. The framework starts with a preprocessing step, where the lung fields are extracted from the CT scans and the background value is set to $0 \mathrm{HU}$. First, an affine transform is applied to correct for global deformations. Then a series of multi-resolution B-Spline transforms with decreasing grid resolution is applied to the affinely registered images. Each transform was optimized using the stochastic gradient descent method.

Finally, the moving image is deformed based on the obtained deformation field. To minimize the intensity differences in the fixed and moving images caused by the difference in respiratory level, the intensities of the deformed image are adjusted with respect to the Jacobian determinant of the deformation field as proposed in [6]. The baseline image $I_{1}$ was set as the fixed image, and the four follow up images $I_{2 . .5}$ were set as the moving images in the registration framework. 


\subsection{Local Image Dissimilarity}

The registration results in dense spatial correspondence, but small misregistrations in the order of $1 \mathrm{~mm}$ remain. To minimize the impact of the misregistration, we propose to compare points in the different images using a simplified version of locally orderless images (LOI) 9], where the inner, outer and tonal scales are fixed. A local histogram is constructed using a weighted window function centered around a point $x_{0}$. Given an image $I\left(x_{0}, \sigma\right)$ that is observed under the fixed inner scale $\sigma$, the LOI at a point $x_{0}$ is defined as follows:

$$
h_{I}\left(i ; x_{0}, \alpha\right)=\frac{1}{(\sqrt{2 \pi} \alpha)^{3}} \int_{0}^{x} A\left(x, x_{0}, \alpha\right) e^{-(I(x, \sigma)-i)^{2}} d x,
$$

where $\alpha$ is the outer scale, which corresponds to the size of the window function $A\left(x, x_{0}, \alpha\right)$ and $i$ is an intensity value. Later in text we denote the histogram using a shorter notation $h=h_{I}\left(i ; x_{0}, \alpha\right)$.

In order to capture different features, in addition to the original image $I$ $(\sigma=0)$, LOIs are also computed from the blurred image and the gradient magnitude. The feature images are all observed under the same scale, which is achieved by blurring the images using a Gaussian kernel with a standard deviation of $\sigma$.

Given the two histograms $h_{1}(i ; x)$ and $h_{t}(i ; x)$ obtained in the same anatomical point $x$ from the two images $I_{1}$ and $I_{t}$ respectively, we compute a set of dissimilarity measures $D\left(I_{1}, I_{t}\right)(x)=\left\{d_{i}\left(h_{1}, h_{t}\right)\right\}$ between the histograms.

In this paper, we use two classes of dissimilarity measures. The first class consists of L1-norm and Kullback-Leibler divergence between the two histograms $d_{1}=\left\|h_{1}-h_{t}\right\|_{L_{1}}, d_{2}=\left\|h_{1}-h_{t}\right\|_{L_{2}}, d_{3}=D_{K L}\left(h_{1}, h_{t}\right)$. In the second class, the dissimilarity between the local histograms is computed as the difference between the individual measures on each of the histograms $d_{i}=m_{i}\left(h_{1}\right)-m_{i}\left(h_{t}\right)$ : the first four moments, the mode, the energy; and the maximum of difference between the cumulative distribution functions of the histograms $d_{n}=\max \left(\operatorname{cdf}\left(h_{1}\right)-\right.$ $\left.c d f\left(h_{t}\right)\right)$ [10.

\subsection{Disease Progression Measure}

Since LOIs have a certain region of influence, it is not required to compare each and every point in the images. Therefore, a sparse representation of the image is used for comparison instead, where comparison is only performed on a fixed number of regions, $N_{s}$, sampled randomly within the image.

For every sample $x_{i}$ we compute the set of dissimilarity measures $D_{I}=$ $D\left(I_{1}, I_{t}\right)$ between the images $I_{1}$ and $I_{t}$, and the filtered versions of the images $D_{G}=D\left(I_{1, \sigma}, I_{t, \sigma}\right), D_{G M}=D\left(\left|\nabla I_{1, \sigma}\right|,\left|\nabla I_{t, \sigma}\right|\right)$. The subscripts $I, G, G M$ denote the original image and response to the Gaussian and Gaussian magnitude filters respectively. Therefore dissimilarity between the two images at the location $x_{i}$ is defined by the dissimilarity vector $\boldsymbol{D}_{1, t}=\left\{D_{I}, D_{G}, D_{G M}\right\}_{1, t}$.

The dissimilarity measures from the first class assess the distance between the corresponding local histograms. The dissimilarity measure from the second 
class assess the change in the histogram characteristics. If the histograms differ, dissimilarity measures from the first class are strictly positive while the dissimilarity from the second class result in both positive and negative values. We are interested in local changes regardless of the sign therefore only the magnitude of the dissimilarity measures is considered. Finally, the measure of local changes $p_{1, t}\left(x_{i}\right)$ at the sample $x_{i}$ between the images $I_{1}$ to $I_{t}$ is computed as the L1-norm of the dissimilarity vector, as follow, $p_{1, t}\left(x_{i}\right)=|| \boldsymbol{D}_{1, t} \|_{L_{1}}$.

\section{Experiments}

\subsection{Data}

We conducted experiments on subjects with Alpha 1-antitrypsin deficiency monitored during a period of 30 months. A total of 27 subjects were included into the experiments. For each subject low-dose CT images were acquired at five time points: at baseline, after 3 , after 12 , after 21 , and after 24 or 30 months. Out of 27 subjects 11 were scanned after 24 months. The scans were acquired using a tube voltage of $140 \mathrm{kVp}$, exposure $40 \mathrm{mAs}$, in-plane resolution $0.78 \mathrm{~mm}$ and slice thickness $2 \mathrm{~mm}$ without overlap.

Lung function tests were acquired along with the CT scans, of which we used the forced expiratory volume in 1 second $\left(\mathrm{FEV}_{1}\right)$. At baseline all the patients performed lung function tests and average $\mathrm{FEV}_{1}$ for all the subjects was $1.54 \pm$ $0.68 \mathrm{~L}$, and TLC was $8.02 \pm 1.57 \mathrm{~L}$, the ratio $\mathrm{FEV}_{1} / \mathrm{TLC}$ was $20.27 \pm 10.38$ $\%$. For the last visit there are 2 missing lung function tests, and the average over the remaining 25 subjects is $\mathrm{FEV}_{1} 1.29 \pm 0.71 \mathrm{~L}$, TLC $7.45 \pm 2.51$ and ratio $\mathrm{FEV}_{1} / \mathrm{TLC} 17.93 \pm 9.04 \%$.

\subsection{Measuring Local Emphysema Progression}

The four follow up CT scans $I_{2}, I_{3}, I_{4}, I_{5}$ were first registered to the baseline image $I_{1}$. The segmented lung fields from the baseline image $I_{1}$ were eroded with a structuring element of size of $3 \times 3 \times 3$ voxels and $N_{s}=2000$ positions were randomly sampled from the eroded lung fields. In our experiments we chose the Gaussian scale of the filters $\sigma=1$ voxel. The radius of the aperture function $A$ was set to $\alpha=20$ voxels, and the weights were truncated at $3 \alpha$ radius. For the intensity-based histograms the bin width was set to $1 \mathrm{HU}$ resulting in 1000 bins in total in the intensity range from -1000 to $0 \mathrm{HU}$. For the histograms of the filtered images, the number of bins was set to 1000 and the bin edges were placed uniformly covering the full range of filter responses.

Within a 3 month period changes are expected to be relatively small, therefore the dissimilarities observed in this period reflects mostly image dissimilarity caused by misregistration and interpolation. From this pair of images we obtained the mean and the standard deviation of the dissimilarity vector $\boldsymbol{D}_{1,2}$. Further we normalized all the dissimilarity vectors $\boldsymbol{D}_{1, t=2,3,4,5}$ with respect to the obtained mean and standard deviation and then computed the corresponding progression measures $p_{1, t=2,3,4,5}$. 


\section{Results}

Table 1 reports the summary of the conventional emphysema progression measurements, the decline in $\mathrm{FEV}_{1}\left(\Delta \mathrm{FEV}_{1}\right.$ in $\left.\mathrm{L}\right)$ and increase of relative area below the $950 \mathrm{HU}(\Delta \mathrm{RA} 950$ in $[\%])$. The conventional measures were compared with the proposed feature-based disease progression measures. Disease progression measure (PM) on a subject level was computed as the average of dissimilarity measures for all spatial locations. We tested the complete set of dissimilarities (PM (all)) and only Kullback-Leibler divergence between the local histograms of the smoothed images as the local dissimilarity measures (PM (KL)) or only local increase in RA950HU (PM (loc $\Delta$ RA950)). Table 1 presents the average disease progression measures for all consecutive follow up visits. A time trend analysis was performed for the disease progression measurements using a linear mixed model with the time between the baseline and a follow up visit as fixed effect. For the FEV1 we did not conducted time trend analysis because 9 out of 27 subjects had missing FEV1 at least at one of the visits. The t-values are reported in the Table 1. Additionally, correlation coefficients between the progression measured at the last visit by the proposed methods and by conventional emphysema score and lung function are presented in the Table 1 .

Table 1. Summary of the disease progression measures. Left part presents the average of the progression measures over all subjects for the follow up visits and the t-value of the time-trend analysis. The correlation coefficients between the progression measures obtained from the last visit with p-values in brackets are presented in the right part of the table.

\begin{tabular}{|c|c|c|c|c|c|c|c|}
\hline \multicolumn{5}{|c|}{ Average Progression } & \multirow{2}{*}{$\begin{array}{c}\text { Time-trend } \\
\text { t-value }\end{array}$} & \multicolumn{2}{|c|}{ Correlation coefficients } \\
\hline \# mnths & 3 & 12 & 21 & $30(24)$ & & $\Delta \mathrm{RA} 950$ & $\Delta \mathrm{FEV}_{1}$ \\
\hline$\Delta \mathrm{FEV}_{1}$ & -0.03 & 0.01 & -0.01 & -0.13 & & & \\
\hline$\Delta \mathrm{RA} 950$ & -1.27 & 0.08 & 1.33 & 1.91 & 6.37 & & $-0.18(0.48)$ \\
\hline PM (all) & 0.75 & 0.76 & 0.89 & 0.93 & 3.09 & $0.51(0.007)$ & $0.11(0.59)$ \\
\hline $\mathrm{PM}(\mathrm{KL})$ & 0.25 & 0.27 & 0.30 & 0.31 & 7.80 & $0.45(0.02)$ & $-0.18(0.39)$ \\
\hline PM (loc $\Delta$ RA950) & 0.0 & 0.46 & 0.77 & 1.03 & 8.80 & $\| 0.87(<0.001)$ & $0.11(0.59)$ \\
\hline
\end{tabular}

Fig 1 shows samples locations, indicated with circles, overlaying on the 2Dslices extracted from the registered follow up images. Radius of a circle in the follow up images is proportional to the dissimilarity measure computed from the complete set of dissimilarities. Each row represents different subjects.

In order to investigate local consistency of the local disease progression measures, we tested the simple hypothesis that samples with dissimilarity measure above a threshold $T$ at the previous follow up visit should not decrease the dissimilarity measure in the consecutive visits. The threshold on the dissimilarity measure was selected based on the 25 th- and 75 th- percentiles, $p_{25}$ and $p_{75}$, of the dissimilarity measures after the 3 months follow up visit, $T=p_{75}+1.5\left(p_{75}-p_{25}\right)$ which corresponds to $\sim 2.7$ standard deviations. The total number of samples with dissimilarity above the threshold $T$ and the relative percentage of those 

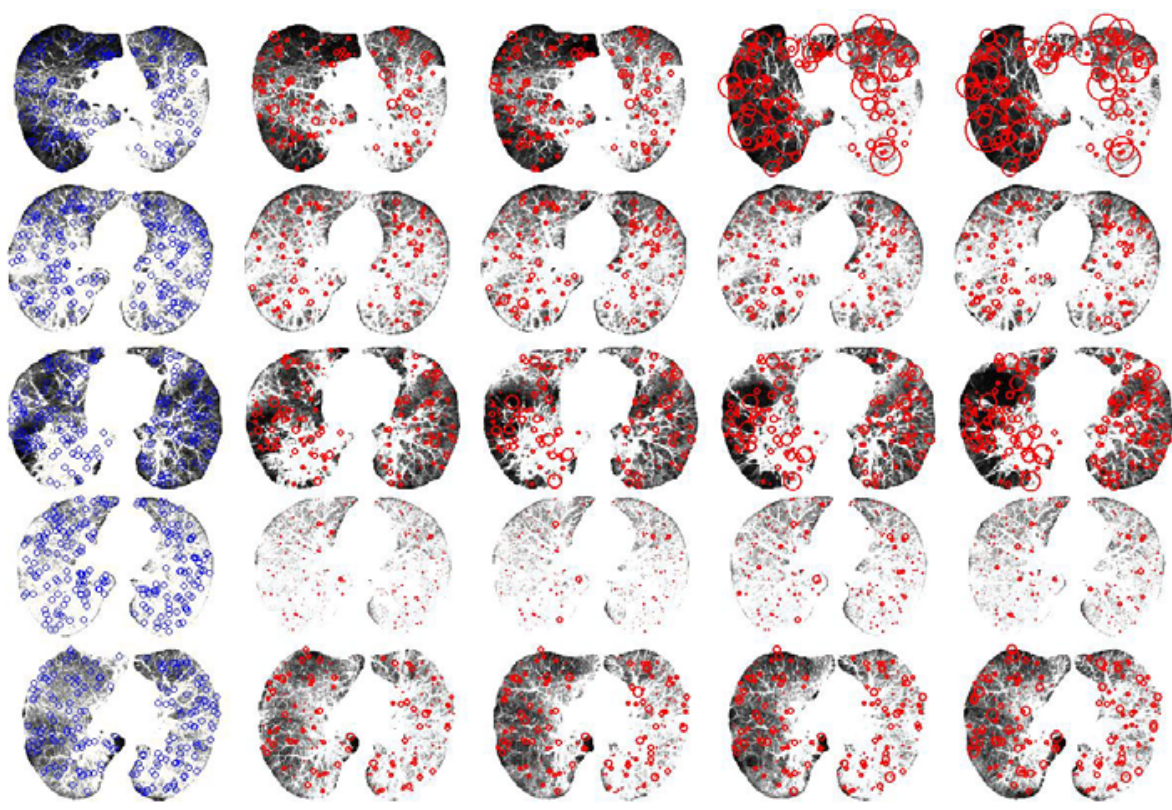

Fig. 1. Rows show mean intensity projection over a stack of 9 sequential slices, selected from different volumetric images. The left most column shows slices extracted from the baseline image, the remaining columns show corresponding slices extracted from the registered 3, 12, 21 and 30(24) months follow up visits, respectively from left to right. All the slices are displayed in the intensity range [-1000,-900]HU. Locations of the random samples (blue and red markers) in the corresponding stack were projected to the image slice. In the follow up images the marker size is proportional to the local dissimilarity measure obtained from the complete set of dissimilarities.

Table 2. Comparison of the local dissimilarity measures. Table presents the overall percentage of samples with dissimilarity measure above the threshold $T$; in brackets the relative percentage of sampled which increased or preserved the dissimilarity measure above the threshold in all the successive follow up scans.

\begin{tabular}{|l|cccc|}
\hline \multicolumn{4}{|c|}{ Overall percentage [\%] } & (confirmed [\%]) \\
\multicolumn{1}{|c|}{ \# mnths } & 3 & 12 & 21 & $30(24)$ \\
\hline PM (all) & $5.24(41.61)$ & $5.68(61.48)$ & $9.01(75.87)$ & 10.49 \\
PM (KL) & $1.19(38.73)$ & $1.75(56.07)$ & $3.72(78.12)$ & 4.93 \\
PM (loc $\Delta$ RA950) & $2.86(9.07)$ & $4.38(33.94)$ & $8.88(60.54)$ & 11.48 \\
\hline
\end{tabular}

samples that increase or preserve the same dissimilarity measure in all the successive visits is reported in the Table 2 .

Examples of the samples with disease progression measure above the threshold $T$ are presented in the Fig.2. Left plot in Fig.2 shows a subject where most of the samples with the large dissimilarity measure after 3 months were confirmed with all the consecutive follow up scans. Right plot in Fig.2, where the samples did not show consistent dissimilarity measure over time. 

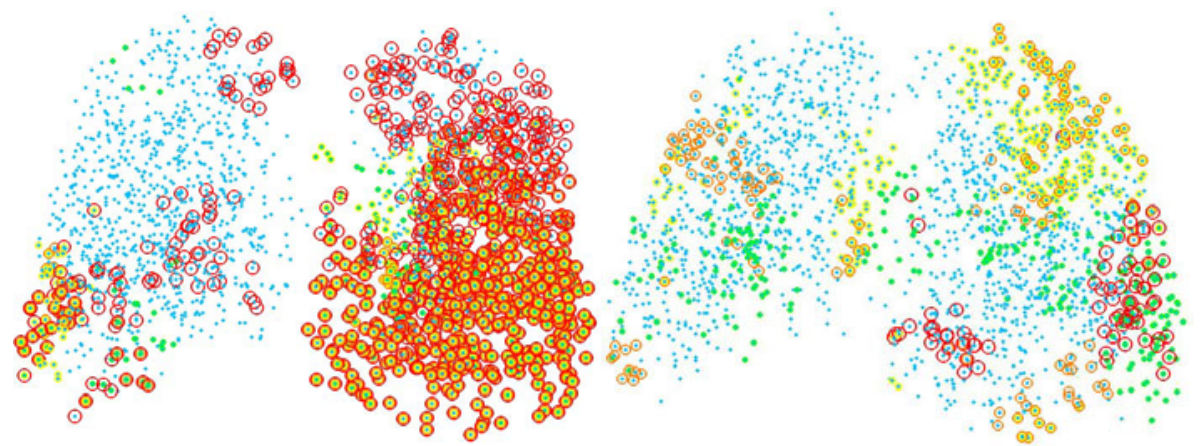

Fig. 2. All selected random samples marked in blue color. Locations with the significantly large dissimilarity measure obtained from the complete set of features at the 3 , $12,21,30(24)$ months follow up visits are indicated in green, yellow, orange and red markers respectively.

\section{Discussion}

In this paper we presented a framework for detection of local emphysema progression. The overall disease progression measure showed significant correlation $(p<0.01)$ with increase in the standard CT score, the relative area below $-950 \mathrm{HU}$, between the baseline and the last follow up visit. The correlation with the decline in $\mathrm{FEV}_{1}$ was not significant for neither the proposed measures nor for the standard CT score. In our dataset the average $\mathrm{FEV}_{1}$ at baseline was very low, indicating the severity of emphysema already at the baseline visit. This can explain the lack of sensitivity to disease progression of the $\mathrm{FEV}_{1}$ measurement.

We analyzed time trend based on the conventional emphysema measurements and the proposed dissimilarity-based measurements. The time trend was approximately equally significant for the conventional RA950 disease progression measure, local increase in RA950 and the measure derived from the KullbackLeibler divergence between local histograms of the smoothed images. The time trend was less significant for the measurement obtained from the complete set of dissimilarities. One of the possible explanations could be sensitivity of the particular dissimilarity measure to the change in image appearance not related to the emphysema progression, for example inflammation or change in local topology like collapsing or appearing bullae. Another possible explanation could be in the construction of the overall combined disease progression score from the complete vector of dissimilarities.

The current drawback of the proposed method is the simplification of the complete dissimilarity vector by its norm. The emphysema is usually characterized by the destruction of the lung tissue thus decreasing image intensities, while inflammation should result in increase of image intensities. In the current framework the two phenomena could result in equal dissimilarity measures. The specific dissimilarity measures such as difference in means of the local histograms is capable of differentiating between the two processes, therefore a 
careful investigation of the dissimilarity measures should be done. Furthermore an automatic classification approach could be adapted for this problem, where samples from the image pairs with the 3 months follow up scan represent stable group while samples from the image pairs with the 30(24) months follow up scan represent progressed group.

To conclude, we proposed a method for estimating local disease progression. Results suggested that emphysema progression can be detected before the tissue intensity decreases below the standard CT threshold of -950HU.

Acknowledgments. This work is financially supported by the Danish Council for Strategic Research under the Programme Commission for Nanoscience and Technology, Biotechnology and IT (NABIIT), the Netherlands Organisation for Scientific Research (NWO), and AstraZeneca, Lund, Sweden.

\section{References}

1. Rabe, K., et al.: Global strategy for the diagnosis, management, and prevention of chronic obstructive pulmonary disease gold executive summary. Am. J. Respir. Crit. Care. Med. 176, 532-555 (2007)

2. Snider, G., Kleinerman, J., Thurlbeck, W., Bengali, Z.: The definition of epmhysema: report of a National Heart, Lung, and Blood Insitute, division of lung diseases workshop. Am. Rev. Resp. Dis. 132, 182-185 (1985)

3. Gietema, H., Schilham, A., van Ginneken, B., van Klaveren, R., Lammers, J., Prokop, M.: Monitoring of smoking-induced emphysema with CT in a lung cancer screening setting: Detection of real increase in extent of emphysema. Radiology 244(3), 890-897 (2007)

4. Dirksen, A., Piitulainen, E., Parr, D., Deng, C., Wencker, M., Stockley, S.S.R.: Exploring the role of CT densitometry: a randomised study of augmented therapy in $\alpha_{1}$-antitrypsin deficiency. Eur. Respir. J. 33, 1345-1353 (2009)

5. Staring, M., Bakker, M., Shamonin, D., Stolk, J., Reiber, J., Stoel, B.: Towards local estimation of emphysema progression using image registration. In: Pluim, J., Dawant, B. (eds.) SPIE (Medical Imaging). Proceedings of SPIE, vol. 7259 (February 2009)

6. Gorbunova, V., Lo, P., Ashraf, H., Dirksen, A., Nielsen, M., de Bruijne, M.: Weight preserving image registration for monitoring disease progression in lung CT. In: Metaxas, D., Axel, L., Fichtinger, G., Székely, G. (eds.) MICCAI 2008, Part II. LNCS, vol. 5242, pp. 863-870. Springer, Heidelberg (2008)

7. Sørensen, L., Lo, P., Ashraf, H., Sporring, J., Nielsen, M., de Bruijne, M.: Learning COPD sensitive filters in pulmonary CT. In: Yang, G.-Z., Hawkes, D., Rueckert, D., Noble, A., Taylor, C. (eds.) MICCAI 2009. LNCS, vol. 5762, pp. 699-706. Springer, Heidelberg (2009)

8. Arzhaeva, Y., Prokop, M., Murphy, K., van Rikxoort, E., de Jong, P., Gietema, H., Viergever, M., van Ginneken, B.: Automated estimation of progression of interstitial lung disease in CT images. Medical Physics 37(1), 63-73 (2010)

9. Koenderink, J.J., Van Doorn, A.J.: The structure of locally orderless images. Int. J. Comput. Vision 31(2-3), 159-168 (1999)

10. van Ginneken, B., ter Haar Romeny, B.M.: Multi-scale texture classification from generalized locally orderless images. Pattern Recognition 36(4), 899-911 (2003) 